\title{
Analysis and Dynamics of Extended Atomic Defects in Coalesced WS2 Monolayer Films
}

Danielle Reifsnyder Hickey ${ }^{1}$, Saiphaneendra Bachu ${ }^{2}$, Leixin $\mathrm{Miao}^{3}$ and Nasim Alem ${ }^{4}$

${ }^{1}$ Pennsylvania State University, Pennsylvania, United States, ${ }^{2}$ Pennsylvania State University, United States, ${ }^{3}$ Department of Materials Science and Engineering, The Pennsylvania State University, University Park, Pennsylvania, United States, ${ }^{4}$ Pennsylvania State University, Washington, District of Columbia, United States

Monolayer transition metal dichalcogenides (TMDs) hold great promise for optoelectronic devices, but their potential for successful application is limited by the various types of atomic defects that can exist [1]. Such defects can be introduced during growth, processing, and subsequent analysis, and their structures can be understood using aberration-corrected scanning transmission electron microscopy (STEM).

In monolayer TMD films, line defects such as grain boundaries result from the merging of misoriented islands during growth [2]. Optimization of growth parameters can potentially eliminate these grain boundaries, and a recent advance in film growth by metal-organic chemical vapor deposition has produced 2 " wafer-scale single-orientation monolayer $\mathrm{WS}_{2}$ films on sapphire substrates [3]. However, annular darkfield (ADF-) STEM imaging reveals that these films still contain defect arrays that result from the coalescence of grains.

Two types of linear defect arrays will be explored in this presentation. First, where oriented $\mathrm{WS}_{2}$ grains merge with a sub-unit cell offset, translational grain boundaries exist, which are analyzed using ADFSTEM imaging [4]. ReaxFF reactive force field molecular dynamics (MD) simulations show that their edge morphologies correspond to relatively fast growth, and dark-field (DF-) TEM also shows that these grain boundaries form networks throughout the monolayer films. Statistical analysis of the edge orientations shows their preferred angular distribution relative to the zigzag edge of $\mathrm{WS}_{2}$, which is related to the structure and periodicity of atomic units along the boundary.

Second, where oriented $\mathrm{WS}_{2}$ grains merge in registry, $\mathrm{W}$-vacancy arrays exist [5]. The experimentally observed morphologies of these metal-vacancy arrays will be discussed and explained in the context of their growth mechanisms. ReaxFF MD simulations support that the vacancy arrays form during film coalescence because of the steric and catalytic effects of the precursor molecules, substrate, and a nearby $\mathrm{WS}_{2}$ edge. Through this framework, mechanisms for different morphologies of $\mathrm{W}$-vacancy arrays will be discussed.

Finally, the in situ electron beam-induced atomic-scale dynamics of defect arrays will be explored. The existence of defect arrays in TMD films highlights the need to engineer film growth and defect formation/annihilation processes. In this case, the in situ atomic transformations that occur are able to provide valuable insights into the process of grain coalescence.

The authors gratefully acknowledge funding provided by the National Science Foundation (NSF) through the Pennsylvania State University 2D Crystal Consortium-Materials Innovation Platform (2DCC-MIP) under NSF cooperative agreement DMR-1539916. D.R.H, S.B., and N.A. also acknowledge support from the NSF CAREER program (DMR-1654107) and the NSF program EFRI 2-DARE (EFRI-1433378). This 
work utilized resources provided by the NSF-MRSEC-sponsored Materials Characterization Lab at Penn State.
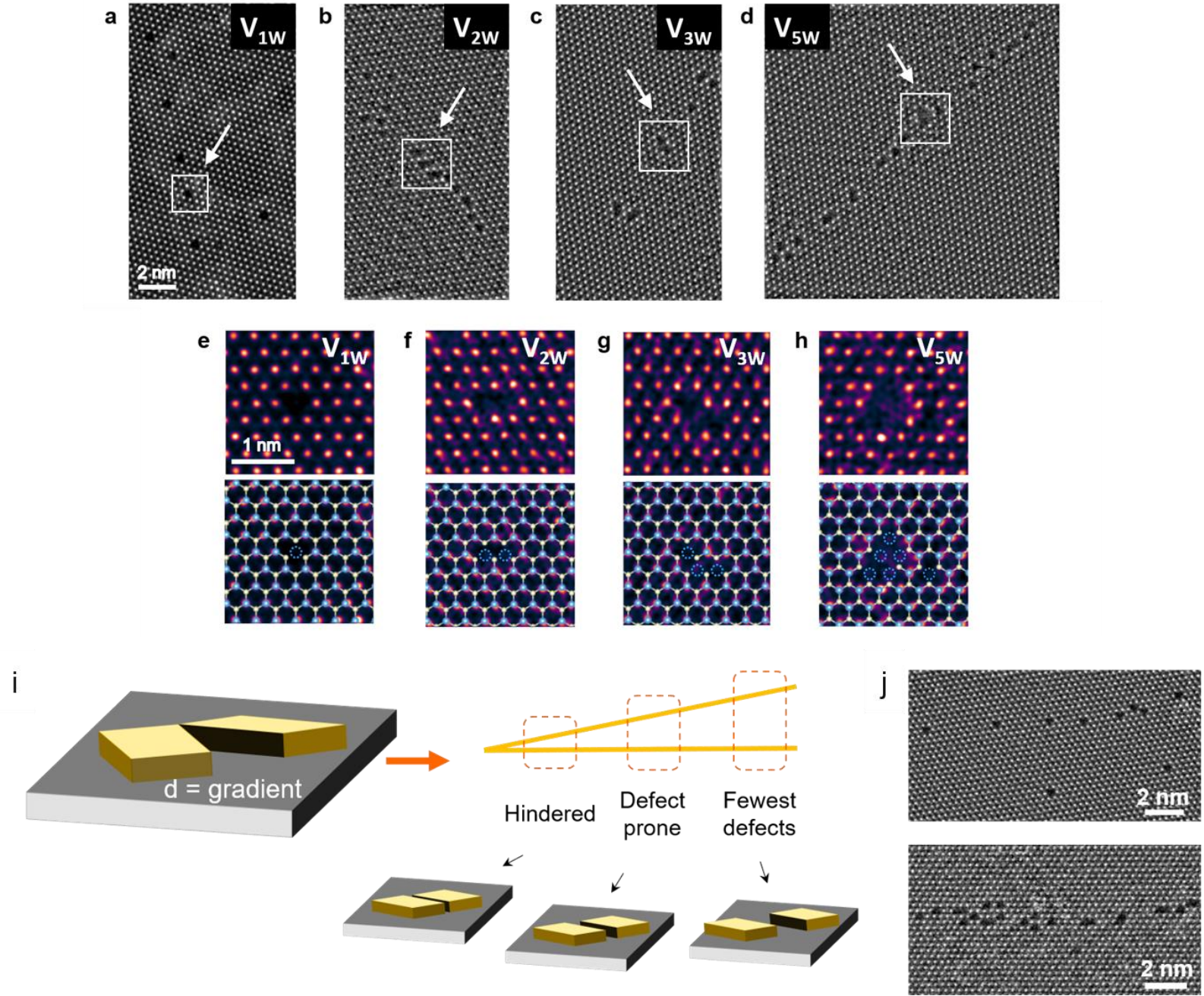

Figure 1. Types of W-vacancy clusters and arrays observed in WS2 films. (a)-(d) ADF-STEM images of vacancy arrays that include various vacancy clusters. White boxes and arrows indicate examples of vacancy clusters. (e)-(h) ADF-STEM images with ball-and-stick atomic models overlaid for (e) single-, (f) double-, (g) triple-, and (h) quintuple-W vacancy clusters (for the models, $\mathrm{W}=$ blue and $\mathrm{S}=$ yellow). (i) Schematic of an angled channel and how the reaction varies with location on the gradient. (j) Examples of ADF-STEM images of an array of aligned point defects and an intermediate cluster array that can be formed by angled channels. Figures 4 and 5 reproduced with permission from ref. [5]. Copyright (2021) by the Institute of Physics.

\section{References}

[1] H. Cai et al., Nano Res. (2020). https://doi.org/10.1007/s12274-020-3047-7

[2] W. Yao et al., ACS Nano 14 (2020) 9320.

[3] M. Chubarov et al., ACS Nano ASAP.

[4] D. Reifsnyder Hickey et al., arXiv:2006.11668 [cond-mat.mtrl-sci] (2020).

[5] D. Reifsnyder Hickey et al., 2D Mater. 8 (2021) 011003. 